\title{
Bibliografía y referencias
}

Alfaro, A.L., Alpízar, M., Morales, Y., Ramírez O., E Salas, O. (2013). La formación inicial y continua de docentes de Matemáticas en Costa Rica. Manuscrito sin publicar. Red de Educación Matemática de América Central y el Caribe.

Arboleda, L.C. (2013). Entrevista personal, en abril.

Artigue, M. (2011). La Educación Matemática como un campo de investigación y como un campo de práctica: Resultados, Desafíos. Conferencia presentada en la XIII Conferencia Interamericana de Educación Matemática, junio 2011, Recife, Brasil. Recuperado el 9 de marzo del 2012 de http://www.gente.eti.br/lematec/CDS/XIIICIAEM/?info _ type=home\& lang_ user=br

Artigue, M. \& Houdement, C. (2007). Problem solving in France: didactic and curricular perspectives. ZDM Mathematics Education 39: 365-382. DOI 10.1007/s11858-007-0048-x

Artigue, M., Douady, R., Moreno, L. \& Gómez, P. (Eds.).(1995). Ingeniería didáctica en Educación Matemática. México: Grupo Editorial Iberoamérica.

Australian Council for Educational Research (2011). PISA 2009: Plus Results. Recuperado de https://mypisa.acer.edu.au/images/mypisadoc/acer_ pisa 2009+ international_ 1.pdf

Ball, D., Hill, H. \& Bass, H. (2005). Knowing mathematics for teaching. Who knows mathematics well enough for teach third grade, and how can we decide? American Educator. 29 (3), 14-17, 20-22, 23-46.

Banco Mundial. (2009, junio). Competitividad en Costa Rica. Recuperado de http://site resources.worldbank.org/INTCOSTARICAINSPANISH/Resources/CostaRicaCompetiti veness.pdf

Barber, M. \& Mourshed, M. (2007). How the Worlds Best-Performing School Systems Come Out On Top, McKinsey \& Company, Social Sector Office. http://www.mckinsey.com/ clientservice/social_sector/our_practices/education/knowledge_highlights/best_performing_school.aspx

Bauersfeld, H. (1994). "Language games" in the mathematics classroom: their function and their effects, en P. Cobb \& H. Bauersfeld (Eds.). The emergence of mathematical meaning: Interaction in classroom cultures. Hillsdale, N. J.: Lawrence Erlbaum Associates.

Brousseau, G. (1998). Theory of Didactical Situations in Mathematics. Dordrecht, The Nedherlands: Kluwer.

Brousseau, G. (2006). Mathematics, didactical engineering and observation. Conferencia presentada en el PME 30, Praga, Julio 2006. Descargado de http://www.math. washington.edu /*warfield/ Didactique.html.

Cai, J. \& Lester, F. (2010). Why is Teaching With Problem Solving Important to Student Learning? NCTM: Reston. A Research Brief enviado el 8 de abril del 2010.

Chaves Esquivel, E. (2007). Inconsistencia entre los programas de estudio y la realidad en el aula en la enseñanza de la estadística de secundaria. Actualidades Educativas en Educación, 7 (3), Recuperado de http://revista.inie.ucr.ac.cr/. 
Chaves Esquivel, E., Castillo, M., Chaves Barboza, E., Fonseca, J. E Loría R. (2010). La enseñanza de las Matemáticas en la secundaria costarricense: entre la realidad y la utopía. Ponencia preparada para el Tercer Informe Estado de la Educación. San José, Programa Estado de la Nación.

Clarke, D., Emanuelsson, J., Jablonka, E. \& Mok, I. (Eds.). (2006). Making connection: Comparing mathematics classrooms around the world. The Netherlands: Sense Publishers.

Clarke, D., Keitel, C. \& Shimizu, Y. (Eds.). (2006). Mathematics classrooms in twelve countries: the insider's perspective. The Netherlands: Sense Publishers.

Cobb, P, Gravemeijer, K., \& Yackel, E. (2011). Chapter 6. Introduction. En E. Yackel, K. Giravemeijer, E A. Sfard, A. Eds.). A Journey in Mathematics Education Research. Insights form the Work of Paul Cobb. Dordrecht, The Nedherlands: Springer.

Cobb, P. (1983). The Constructivist Researcher as Teacher and Model Builder. Journal for Research in Mathematics Education, 14, (83-94).

Cobb, P. (1994). Where is the mind? Constructivist and sociocultural perspectives on mathematical development. Educational Researcher, 23(7), 13-20.

Cobb, P. (2011). Chapter 2. Introduction. En E. Yackel, K. Gravemeijer, \& A. Sfard. (Eds.). A Journey in Mathematics Education Research. Insights form the Work of Paul Cobb. Dordrecht, The Nedherlands: Springer.

Cobb, P. \& Bauersfeld, H. (1994). The emergence of mathematical meaning: Interaction in classroom cultures. Hillsdale, N. J.: Lawrence Erlbaum Associates.

Confrey, J. \& Kazak, S. (2006). A thirty-year reflection on constructivism in mathematics education in PME. En A. Gutiérrez \& P. Boero, Handbook of Research on the Psychology of Mathematics Education. Rotterdam, The Nedherlands: Sense Publishers, (pp. 305-345).

D'Ambrosio, U. (1997). Ethnomathematics and its address in the history and pedagogy of mathematics. En Powell, A. B. \& Frankenstein, M. (Eds.), Ethnomathematics. Challenging Eurocentrism in Mathematics Education, pp.13-24. Albany NY: State University of New York Press.

D'Ambrosio, U. (2007). Peace, social justice and ethnomathematics. The Montana Mathematics Enthusiast, 1:25-34.

D’Ambrosio, U. (2008). Etnomatemática. Eslabón entre las tradiciones y la modernidad. México : Limusa.

Downes, S. (2012). Connectivism and Connective Knowledge: essays on meaning and learning networks. National Research Council Canada, http://www.downes.ca/files/ books/ Connective_ Knowledge-19May2012.pdf. Citado por Ramírez Vega (2013b).

Dubinsky, E. (1992). Reflective abstraction in advanced mathematical thinking. En D. Tall (Ed.), Advanced Mathematical Thinking (pp. 95-124). Dordrecht: Kluwer Academic Publishers.

English, L., Lesh, R., \& Fennewald, T. (2008). Future directions and perspectives for problem solving research and curriculum development. Presentado al 11 th International Congress on Mathematical Education, Julio 6-13 de 2008 en Monterrey, México.

Ernest, P. (2011). The psychology of learning mathematics: The cognitive, affective and contextual domains of mathematics education. Saarbrûcken, Alemania: Lambert Academic Publishing.

Even, R. \& Ball, D. (2008). The professional education and development of teachers of mathematics: The 15th ICMI Study. New York: Springer. Citado por Artigue (2011). 
Figueroa, N. (2013, 18 de abril). [Carta enviada a Ruth de la Asunción Romero, Vicerrectora de Vida Estudiantil de la Universidad de Costa Rica]. Copia en la Escuela de Matemática de la Universidad de Costa Rica. Costa Rica.

Figueroa, N. E Jiménez, K. (2010). Primer informe de resultados. Examen de Diagnóstico en Matemática, DiMa. Recuperado de http://www.diagnostico.emate.ucr.ac.cr/sites/ diagnostico.emate.ucr.ac.cr/files/PrimerlnformeDiMa2010.pdf

Freudenthal, H. (1973). Mathematics as an educational task. Dordrecht, The Nedherlands: Reidel

Freudenthal, H. (1983). Didactical phenomenology of mathematical structures. Dordrecht, The Nedherlands: Reidel

Freudenthal, H. (1991). Revisiting mathematics education: China lectures, Dordrecht: Kluwer Academic Publ.

Fullan, M. (2008). The six secrets of change. Descargado de http://www.michaelfullan.ca/ images/handouts/2008SixSecretsofChangeKeynoteA4.pdf

Gravemeijer, K. (2010). Preamble: from models to modeling. En Gravemeijer, K., Lehrer, R., van Oers, B. \& Verschaffel, L. (Eds.). Symbolizing, Modeling and Tool Use in Mathematics Education. Dordrecht, The Nedherlands: Kluwer Academy Press. (pp.7-22).

Herman, R. L. (2012). The MOOCs Are Coming. The Journal of Effective Teaching, 12(2), 1-3. Citado por Ramírez Vega (2013b).

Hernández, L. (2013). UVA: Unidades Virtuales de Aprendizaje. Manuscrito sin publicar.

Hiebert, J.; Gallimore, R.; Garnier, H.; Givvin, K.; Hollingsworth, H.; Jacobs, J.; Chui, A.; Wearne, D.; Smith, M.; Kersting, N.; Manaster, A.; Tseng, E.; Etterbeek, W.; Manaster, C.; Cionzales, P. \& Stigler, J. (2003). Teaching Mathematics in Seven Countries. Results From the TIMSS 1999 Video Study. EUA: US Department of Education, National Center for Education Statistics.

Hino, K. (2007). Toward the problem-centered classroom: trends in mathematical problem solving in Japan. ZDM Mathematics Education, 39:503-514.

Isoda, M., Stephens, M., Ohara, Y. \& Miyakawa, T. (2007). Japanese Lesson Study in Mathematics. Its impact, diversity and potential for educational improvement. Singapore: Word Scientific. Citado por Artigue (2011).

Jablonka, E. \& Bergsten, C. (2010). Theorising in mathematics education research: differences in modes and quality. Nordic Studies in Mathematics Education, 15 (1), 25-52.

Kilpatrick, J. (1987). What constructivism might be in mathematics education. En J. C. Bergeron, N. Herscovics \& C. Kieran (Eds.), Proceedings of the Eleventh Conference of the International Group for the Psychology of Mathematics Education (pp. 2-27). Montreal: University of Montreal.

Lesh, R. Er Doerr, H. (2008). En What Ways Does a Models and Modeling Perspective More Beyond Constructivism? En R. Lesh y D. Doerr (Eds.): Beyond Constructivism. Models and Modeling Perspectives on Mathematics Problem Solving. Learning and Teaching. Mahwah, NJ, EUA: Lawrence Erlbaum Associates Publishers.

Lesh, R. E Doerr, H. M. (Eds.). (2003). Beyond constructivism: Models and modeling perspectives on mathematics problem solving, learning and teaching. Mahwah, New Jersey: Lawrence Erlbaum Associates, Inc.

Ministerio de Educación Pública de Costa Rica (1995a). Programa de estudios. Primer ciclo. Matemáticas. Costa Rica: autor. 
Ministerio de Educación Pública de Costa Rica (1995b). Programa de estudios. Segundo ciclo. Matemáticas. Costa Rica: autor.

Ministerio de Educación Pública de Costa Rica (1995c). Programa de estudios. Tercer ciclo. Matemáticas. Costa Rica: autor.

Ministerio de Educación Pública de Costa Rica (1996). Programa de estudios. Educación Diversificada. Matemáticas. Costa Rica: autor.

Ministerio de Educación Pública de Costa Rica (2001a). Programa de estudios. Educación Diversificada. Matemáticas. Costa Rica: autor.

Ministerio de Educación Pública de Costa Rica (2001b). Programa de estudios. Tercer ciclo. Matemática. Costa Rica: autor.

Ministerio de Educación Pública de Costa Rica (2005a). Programa de estudios. Educación Diversificada. Matemáticas. Costa Rica: autor.

Ministerio de Educación Pública de Costa Rica (2005b). Programa de estudios. Tercer ciclo. Matemáticas. Costa Rica: autor.

Ministerio de Educación Pública de Costa Rica (2012). Programas de Estudio Matemáticas. Educación General Básica y Ciclo Diversificado. Costa Rica: autor.

Ministerio de Educación Pública de Costa Rica (2013a). Página web oficial. http://www.mep.go.cr/ CSE/informacion.aspx

Ministerio de Educación Pública de Costa Rica, Departamento de Evaluación (2009). Reglamento de Evaluación de los aprendizajes. Costa Rica: autor.

Ministerio de Educación Pública, Proyecto Reforma de la Educación Matemática en Costa Rica (2013b). René Descartes y la Geometría Analítica. San José, Costa Rica: autor.

Ministerio de Educación Pública, Proyecto Reforma de la Educación Matemática en Costa Rica (2013c). Unidad Virtual de Aprendizaje: El uso de software de geometría dinámica en la educación secundaria. Costa Rica: autor.

Ministry of Education of Singapore (2006a). Mathematics syllabus primary: Singapore. Recuperado de http://www.moe.gov.sg/education/syllabuses/sciences.

Ministry of Education of Singapore (2006b). Mathematics syllabus secondary: Singapore. Recuperado de http://www.moe.gov.sg/education/syllabuses/sciences

Mourshed, M., Chijioke, C. \& Barber, M. (2010). How the world's most improved school systems keep getting better, McKinsey \& Company. Descargado del http://www.mckinsey.com/ clientservice/socialsector/resources/pdf/Worlds_School_Systems_Final.pdf

Nagasaki, E. (1990). Problem solving. En Sin Sansu Kyoiku Kenkyukai (Ed.). Sansu kyoiku no kiso riron (Basic theory of elementary mathematics education), pp. 134-146. Tokyo: Toyokan. Citado por Hino (2007).

National Council of Teachers of Mathematics (1980). An agenda for action: Recommendations for school mathematics in the 1980s. Reston, VA: The Author.

National Council of Teachers of Mathematics (2010a). Making it happen. Reston, VA, Estados Unidos: autor.

National Council of Teachers of Mathematics. (2000). Principles and standards for school mathematics. Reston, VA: autor. 
National Council of Teachers of Mathematics. (2003). Principios y estándares para la Educación Matemática [Traducción de Manuel Fernández Reyes]. Sevilla: Sociedad Andaluza para la Educación Matemática "THALES".

National Council of Teachers of Mathematics. (2006a). Curriculum focal points for prekinder garten through grade 8 mathematics. Reston, VA: autor.

National Council of Teachers of Mathematics. (2007). Perspectives on the design and development of school mathematics curricula. Reston, VA: autor.

National Council of Teachers of Mathematics. (2009). Focus in high school mathematic: Reasoning and sense making. Reston, VA: autor.

National Council of Teachers of Mathematics. (2010b). Mathematics curriculum: Issues, trends, and future directions. Reston, VA: autor.

National Governors Association Center for Best Practices, Council of Chief State School Officers. (2012). Common Core State Standards. Washington D.C: National Governors Association Center for Best Practices, Council of Chief State School Officers. Sitio web http://www.corestandards.org/the-standards Recuperado el 2 de marzo del 2012.

National Research Council of the United States. NRC (2003). How students learn: History, math and science in the classroom. Washington, DC: National Academy Press.

Neubrand, J. (2006). The TIMSS 1995 and 1999 Video Studies. En Leung, F.K.S.; Graf, K.D. \& Lopez-Real, F. (2006) Mathematics Education in Different Cultural Traditions. A comparative Study of East Asia and the West. The 13th ICMI Study. USA: Springer.

OECD. (2003). The PISA 2003 assessment framework - mathematics, reading, science and problem solving knowledge and skills. Paris: OECD.

OECD. (2005). Informe PISA 2003. Aprender para el mundo del mañana. España: Santillana Educación S.L.

OECD. (2006). Assessing scientific, reading and mathematical literacy a framework for PISA 2006. París: autor.

OECD. (2010a). PISA 2012 Mathematics framework. Descargado de http://www.oecd.org/ dataoecd/ 8/38/46961598.pdf el 6 de marzo del 2012.

OECD. (2010b). Pisa 2009 results: What Students Know and Can Do - student performance in reading, mathematics and science [Vol. I]. Recuperado de http://www.oecd.org/pisa/ pisaproduct s/48852548.pdf

Pehkonen, E. (2008). Some background factors for the Finnish PISA results in mathematics. Mediterranean Journal for Research in Mathematics Education, Vol. 7 (1).

Pehkonen, E., Hannula, M. \& Björkqvist, O. (2007). Problem solving as a teaching method in mathematics education En Pehkonen, E., Ahtee, M. \& Lavonen, J. (eds). How Finns Learn Mathematics and Science. Sense Publishers: Rotterdam/Taipai.

Piaget, J. (1950). The Psychology of Intelligence. New York: Routledge.

Piaget, J. (1970). Genetic Epistemology. New York: Columbia University Press.

Piaget, J. (1973). To understand is to invent. New York: Grossman.

Polya, G. (1990). Cómo plantear y resolver problemas. México: Trillas. 
Programa Estado de la Nación. (2008). Estado de la región en desarrollo sostenible, un informe desde Centroamérica y para Centroamérica. San José, Costa Rica: autor.

Programa Estado de la Nación. (2011). Estado de la Educación 3. San José, Costa Rica: Consejo Nacional de Rectores, Programa Estado de la Nación.

Radford, L. (2008). Theories in Mathematics Education. A Brief Inquiry into their Conceptual Differences. Working Paper. Junio 2008. Preparado para el ICMI Survey Team 7. The notion and role of theory in mathematics education research. Recuperado de http://www.laurentian.ca/NR/ rdonlyres/77731A60-1A3E-4168-9D3EF65ADBF37BAD/0/ radfordicmist7.pdf

Ramírez Vega, A. (2013a). Diseño, desarrollo e implementación del curso MA-1404 Cálculo para estudiantes del TEC mediante estrategias de e-learning (Tesis de Licenciatura en Enseñanza de la Matemática asistida por computadora). Instituto Tecnológico de Costa Rica, Costa Rica.

Ramírez Vega, A. (2013b). MOOCs para la capacitación de docentes en los nuevos Programas de Matemática. Manuscrito sin publicar.

Rico, L. \& Lupiáñez, J. (2008). Competencias matemáticas desde una perspectiva curricular. Madrid, España: Alianza Editorial.

Robert, A. \& Rogalski,J. (2002). Le système complexe et cohérent des pratiques des enseignants de mathématiques: une double approche, Revue Canadienne de l'Enseignement des Sciences, des Mathématiques et des Technologies. 2 (4), 505-

Rolf, W.M. E Radford, L. (2011). A Cultural-Historical Perspective on Mathematics Teaching and Learning. Rotterdam: Sense Publishers.

Ruiz, A. (1985a). Implicaciones teórico-filosóficas del Teorema de Giödel en el paradigma racionalista de la reflexión sobre las Matemáticas. Revista de Filosofía de la Universidad de Costa Rica. Vol. XXIII, N. 58, Diciembre 1985, San José, Costa Rica.

Ruiz, A. (1985b). El factor < paradojas> y el factor < Ciödel> en los Fundamentos de la Matemática. Revista de ciencia y tecnología de la Universidad de Costa Rica, Vol. IX (1-2), 97-108 (1985). San José, Costa Rica.

Ruiz, A. (1987). Fundamentos para una nueva actitud en la enseñanza moderna de las Matemáticas Elementales. Boletín de la Sociedade paranaense de matemática. Vol. VIII (1), Junio, Curitiba, Brasil.

Ruiz, A. (1988). El papel de la Filosofía y la Historia en la Enseñanza de las Matemáticas. Memorias de la Segunda Reunión Centroamericana sobre formación de Profesores e Investigadores en Matemática Educativa, Marzo de 1988, Ciudad de Ciuatemala, Cuuatemala

Ruiz, A. (1990a). Matemáticas y filosofía. Estudios logicistas. San José: Editorial de la Universidad de Costa Rica. 
Ruiz, A. (1990b). Matemáticas: una reconstrucción histórico-filosófica para una nueva enseñanza. Publicado en UNESCO. Educación Matemática en las Américas VII (Actas de la VII Conferencia Interamericana de Educación Matemática, celebrada en República Dominicana, 12-16 julio 1987). Republicado en Cuadernos de Investigación y Formación en Educación Matemática. N. 7. Julio 2011. Costa Rica.

Ruiz, A. (1992). Las matemáticas modernas en las Américas, Filosofía de una Reforma, Educación matemática (Revista Iberoamericana de Educación Matemática): Vol. 4, No. 1, abril 1992, México.

Ruiz, A. (1995a). (Editor). Historia de las Matemáticas en Costa Rica. Una introducción. San José, Costa Rica: Edit. UCR, UNA.

Ruiz, A. (1995b). Fundamentos teóricos e históricos de la reforma de los programas de matemáticas en la primaria y secundaria costarricenses en 1995. En Memoria Novena Reunión Centroamericana y del Caribe sobre formación de profesores e investigadores en Matemática Educativa. La Habana, Cuba: agosto de 1995.

Ruiz, A. (1995c). Constructivismo empírico y filosofía de las matemáticas comentario sobre ideas de Kitcher y Ernest. En Memoria Novena Reunión Centroamericana y del Caribe sobre formación de profesores e investigadores en Matemática Educativa. La Habana, Cuba: agosto.

Ruiz, A. (1997). La Educación Matemática en Costa Rica. Un plan de emergencia. Manuscrito en archivo personal.

Ruiz, A. (2000). El desafío de las Matemáticas. Heredia, Costa Rica: EUNA. Versión digital descargada de http://angelruizz.com/

Ruiz, A. (2001). Asuntos de método en la educación matemática. Revista Virtual Matemática, Educación e Internet, Volumen 2, Número 1, Abril, Instituto Tecnológico de Costa Rica (2001). Cartago, Costa Rica. Descargado de: http://http://www.itcr.ac.cr/revistamate

Ruiz, A. (2006). Universalización de la Educación Secundaria y Reforma Educativa, San José, Costa Rica: EUCR-CONARE.

Ruiz, A. (2010). Conocimientos y currículo en la Educación Matemática. Cuadernos de Investigación y Formación en Educación Matemática, 6, Recuperado de http://revistas.ucr.ac.cr/ index.php/cifem/article/view/6925/6611

Ruiz, A. (2011). La lección de matemáticas a través de estudios internacionales con videos. Cuadernos de Investigación y Formación en Educación Matemática, 8, Recuperado de http://revistas. ucr.ac.cr/index.php/cifem/article/view/6950/6636

Ruiz, A. E Barrantes, H. (1991a). La reforma matemática de la década de los sesenta en Costa Rica: aspectos ideológicos. San José, Costa Rica. En Ruiz, A. (editor): Ciencia y tecnología. Cuadernos del pasado y el futuro, San José: Asociación Costarricense de Historia y Filosofía de la Ciencia, Diciembre.

Ruiz, A. \& Barrantes, H. (1991b). Historia de la implantación de las matemáticas modernas en la educación costarricense. En Ruiz, A. (editor): Ciencia y tecnología. Cuadernos del pasado y el futuro, San José: Asociación Costarricense de Historia y Filosofía de la Ciencia, Diciembre. 
Ruiz, A. \& Barrantes, H. (1998). The History of the Inter American Committee of Mathematics Education. Edición bilingüe español e inglés. Academia Colombiana de Ciencias Exactas, Físicas y Naturales, Bogotá, Colombia, 1998 [Con el apoyo de Barry University de los EUA y la International Commission on Mathematical Instruction (ICMI).

Ruiz, A.; Chavarría, J. y Mora, F. (2003). Tendencias y retos de la Educación Matemática en Costa Rica. Revista Uniciencia, Vol. 20, Número 1 (2003). Facultad de Ciencias Exactas y Naturales, Universidad Nacional. Heredia, Costa Rica.

Ruiz, A. E Tsijli, T. (1995). Los programas de matemáticas de 1995 en el tercer ciclo de la educación general básica en Costa Rica: contenidos e instalación. Memoria Novena Reunión Centroamericana y del Caribe sobre formación de profesores e investigadores en Matemática Educativa. La Habana, Cuba: agosto de 1995.

Ruiz, A., Alfaro, A. E Morales, Y. (2003). Un Cuarto de Siglo en la historia de la Historia de las Matemáticas en Costa Rica. Revista Uniciencia. Vol. 20, Número 2, 225-249. Facultad de Ciencias Exactas y Naturales, Universidad Nacional. Heredia, Costa Rica.

Ruiz, A., Barrantes, H. \& Gamboa, R. (2009). Encrucijada en la enseñanza de las Matemáticas: la formación de educadores. Cartago, Costa Rica: Editorial Tecnológica de Costa Rica.

Schoenfeld, A. (1985). Mathematical problem solving. Orlando: Academic Press.

Schoenfeld, A. (2011). How we think. New York: Routledge.

Sensevy, G. \& Mercier A. (2007). Agir ensemble. L'action conjointe du professeur et des élèves. Rennes: Presses Universitaires de Rennes. Citados por Artigue (2011).

Shimizu, Y. (2006). How Do You Conclude Today's Lesson? The Form and Functions of "Matome" in Mathematics Lessons. En Clarke, D; Emanuelsson, J.; Jablonka, E. \& Mok, I. A. C. (Eds.). Making Connections. Comparing Mathematics Classrooms Around The World. The Netherlands: Sense Publishers.

Shimizu, Y. (2007). What are the characteristics of Japanese Lessons Emerged by the International Comparisons? En Isoda, M; Stephens, M.; Ohara, Y. \& Miyakawa, T. Japanese Lesson Study in Mathematics, Singapore: World Publishing Co.

Shimizu, Y. (2009). Characterizing exemplary mathematics instruction in Japanese classrooms from the learner's perspective. ZDM Mathematics Education 41:311-318.

Shulman, L. (1986). Those who understand: Knowledge growth in teaching. Educational Researcher, 15(2), 4-14.

Sierpinska, A. \& Kilpatrick, J. (Eds.). (1998). Mathematics education as a research domain: a search for identity. An ICMI study. Dordrecht, The Netherlands: Kluwer Academic Press.

Soh, C. K. (2008). An Overview of Mathematics Education in Singapore. En Z. Usiskin \& E. Willmore (Eds.). Mathematics curriculum in Pacific Rim countries -China, Japan, Korea and Singapore. USA: IAP Information Age Publishing Inc.

Stanic, G., \& Kilpatrick, J. (1988). Historical Perspectives on Problem Solving in the Mathematics Curriculum. En R. I. Charles \& E. A. Silver (Eds.), The teaching and assessing of mathematical problem solving (pp. 1-22). Reston, VA: National Council of Teachers of Mathematics.

Stigler, J. \& Hiebert, J. (2004). Improving mathematics teaching. Educational Leadership, 6 (5), 12-17.

Stigler, J. W. \& Hiebert, J. (1999). The teaching gap. New York: The Free Press. 
Treffers, A. (1987). Three dimensions: A model of goal and theory description in mathematics instruction - The Wiskobas Project. Dordrecht, The Netherlands: Reidel.

UNESCO. (2008). Segundo Estudio Regional Comparativo y Explicativo (SERCE): Los aprendizajes de los estudiantes de América Latina el Caribe. Recuperado de http://unesdoc.unesco.org/images/0016/001606/160660s.pdf

Valero, P. (2004). Socio-political perspectives on mathematics education. En P. Valero \& R. Zevenbergen (Eds.), Researching the socio-political dimensions of mathematics education: Issues of power in theory and methodology (pp. 5-23). Dordrecht, The Nedherlands: Kluwer Academic Publishers.

Van den Heuvel-Panhuizen, M. (2000). Mathematics education in the Netherlands: A guided tour. Freudenthal Institute Cd-rom for ICME9. Utrecht: Utrecht University.

Von Cilaserfeld, E. (1984). An introduction to radical constructivism. En P. Walzlawick: The invented reality (pp.17-40). New York: Norton.

Von Cilaserfeld, E. (1987). Learning as a constructive activity. En C. Janvier (Ed.). Problems of representation in the teaching and learning of mathematics (pp. 3-189). Hillsdale, N. J.: Erlbaum.

Von Cilaserfeld, E. (1989). Constructivism in Education. En T. Huse \& , T. N. Postlethwaite, The International Encyclopedia of Education Supplementary Volume, Oxford: Pergamon Press.

Von Gilasersfeld, E. (1995). Radical Constructivism: A Way of Knowing and Learning. London: The Falmer Press.

Vygotsky, L. (1978). Mind and society: the development of higher mental processes. Cambridge, Boston: Harvard University Press.

Vygotsky, L. (1986). Thought and language. Cambridge, MA.: MIT, Press.

Vygotsky, L. S. (1987). Collected works (vol. 1). New York: Plenum. 